\title{
Thoracoscopic surgery for tuberculosis
}

\author{
Jie $\mathrm{Ma}^{1, \mathrm{a}}$ \\ ${ }^{1}$ Surgical Department of Nanning Fourth People's Hospital, Nanning, Guangxi, China, 530023 \\ aemail,
}

Keywords: Tuberculosis, Thoracoscopic Surgery

\begin{abstract}
In recent years, the incidence of tuberculosis, the rate of resistance in China was a clear upward trend and the number of cases needing for surgical treatment is increasing. Surgical resection of lung disease can eliminate the source of infection, shorten the course of treatment to reduce the complications of tuberculosis, tuberculosis is an important means of comprehensive prevention and treatment. Video-assisted thoracoscopic surgery for tuberculosis has the advantages of small trauma, little effect on cardiopulmonary function, quick recovery and so on. The operation is more difficult than conventional thoracotomy. As long as the indications of operation are correctly controlled, the perioperative treatment is strengthened and it is satisfactory.
\end{abstract}

\section{Introduction}

China is a high burden of tuberculosis in the world, the number of patients ranked second in the world. The prevalence of tuberculosis is high, the mortality rate is high, the resistance rate is high, the annual rate of decline is low, the drug resistance of tuberculosis is more serious, the initial, acquired and multidrug resistance are present, the non-tuberculosis mycobacterium resistance The rate is also higher [1-2], drug treatment failure or tuberculosis caused by complications requiring surgical treatment of the increasing number of cases. Surgical resection of lung disease can eliminate the source of infection, shorten the course of treatment, reduce tuberculosis complications, is an important means of comprehensive prevention and treatment of tuberculosis [3].

Video-assisted thoracoscopic surgery is a new type of surgical technique which has been introduced in China since the 1990s. It has the advantages of small trauma, small effect on cardiopulmonary function and quick recovery after traditional thoracotomy. In the field of thoracic surgery, Yim [4] 1996 reported the earliest VATS treatment of tuberculosis, in recent years Kerti [5] and Kang [6] also have similar reports. Although VATS has so many advantages, but there is no large-scale domestic use of VATS for tuberculosis diagnosis and treatment of the study. The current literature shows that [7] VATS surgery diagnosis and treatment of tuberculosis in the following three points: 1 . In the noninvasive or minimally invasive means of failure to establish a correct diagnosis. 2. Treatment of multi-drug resistant tuberculosis, 3. Treatment of tuberculosis complications.

\section{Surgical Indications}

Pulmonary tuberculosis thoracoscopic surgery indications and thoracotomy the same, mainly:

Cavity lesions, especially secondary toxoplasmosis: empty tuberculosis is clinically more common. Chronic fibrous cavities of tuberculosis course of persistent, repeated symptoms, sputum positive are the main source of tuberculosis [8]. Thick wall empty caseous necrosis, tuberculosis granulation and fibrous tissue 3-layer structure, the surrounding lung tissue extensive destruction, fibrosis, it is difficult to close naturally, easy to repeated bacteria, hemoptysis, secondary infection, , There is difficult to control the massive hemoptysis, thick-walled empty tubercle granulation layer thick, difficult to enter the cavity to achieve effective sterilization concentration, so the effect of pure medical treatment is not good [9.10]. Therefore, the tuberculosis cavity by the standard anti-TB treatment ineffective or severe hemoptysis and other serious complications should be actively surgical treatment.

Damage to the lungs: tuberculous lung injury refers to the lungs at the same time there are fiber 
holes, cheese lesions, and can be combined with pulmonary atelectasis or bronchiectasis, often accompanied by mixed infection and row of Yin, severe hemoptysis and bronchial pleural fistula The Such as contralateral lung no active lesions, can be actively considered surgery.

Pulmonary tuberculosis ball diameter $>3 \mathrm{~cm}$ or less than $3 \mathrm{~cm}$, but after treatment increased, cannot be excluded lung cancer: reported statistics show that [11,12]: the diagnosis of spherical lesions were not performed before surgery, including pathological report Lung cancer accounted for about 1/4. It has been reported that in 18 cases of lung cancer in 5 cases can be seen accompanied by the presence of tuberculosis cheese pathological changes [13], which may be due to long-term chronic stimulation of tuberculosis caused by epithelial fibrosis.

Tuberculosis complicated with repeated cough blood, medical treatment ineffective [14]. In recent years, bronchial artery embolization has become an effective means of treatment of massive hemoptysis, requiring pneumonectomy in the gradual decline [15]. However, because the method cannot eliminate the lesion, embolization of blood vessels may recycle and may occur neovascularization and other reasons, some cases of long-term poor results, surgery is still the most effective way to treat tuberculosis hemoptysis.

Multidrug-resistant tuberculosis and long-term persistent bacteria: MDR-TB patients are resistant to two or more of the most effective anti-TB drugs at the same time, not only difficult to treat, but also may not be cured and sustained Transmission of multidrug-resistant tuberculosis, increased the possibility of primary MDR-TB. There are high cost of treatment, low cure rate, high mortality rate [16], there are reports in the literature that [17.18] multidrug-resistant tuberculosis cases of surgical indications for active pulmonary tuberculosis lesions in the side of the whole lung or lobe irreversible lesions, Mainly tuberculosis empty, especially with Aspergillus infection, hemoptysis, ipsilateral lung with bronchiectasis, bullous or spontaneous pneumothorax, such patients should take active surgical treatment. Van Leuven et al [19.20] reported that the cure rate of MDR-TB was $80 \%$ or more.

\section{Perioperative Treatment}

Tuberculosis is a chronic infectious disease, but also a systemic wasting disease, surgery is a part of the comprehensive treatment of tuberculosis, in the standard anti-TB treatment based on surgery, to play the greatest role in surgical treatment, access to good long-term results.

Preoperative. General cases can be based on the recent drug sensitivity test results, there are more than three kinds of drugs combined with chemotherapy 2 to 3 months, usually after 6 months of drug treatment, most of the reversible lesions are absorbed and it is more appropriate surgical time [21]. Repeated hemoptysis is best to choose a better patient in the two intervals of surgery, but with emergency surgery indications should be immediately surgery. Suspected malignant lesions, should not be too much emphasis on preoperative anti-TB treatment time, should be surgery as soon as possible, after the diagnosis of tuberculosis, strengthen anti-TB treatment. Surgery of tuberculosis patients, mostly retreatment, pneumonia patients, preoperative sputum to the negative, but not blindly pursue, unlimited prolonged anti-tuberculosis time can increase the risk of drug resistance, affecting the efficacy of surgery. On the timing of MDR-TB surgery, although the literature reported different [22-24], pomerantz and so that in the course of tuberculosis treatment, once found that medical treatment cannot reach the sputum negative, and the lesion or patients cannot tolerate Chemotherapy, it should be timely consideration of surgical treatment. At present, most scholars believe that $[18,25]$ resistant to more than 3 patients after intensive treatment if no improvement in symptoms, sputum is not shoddy as soon as possible surgery. Those who are resistant to 2 should be treated with adequate anti-tuberculosis treatment for less than 3 months.

Postoperative. Postoperative anti-TB treatment still need 6 to 12 months, 18 months of resistance to cure the lesion to eliminate the source of infection. For multidrug-resistant pulmonary tuberculosis after resection of the lung surgery, some scholars believe that $[3,25]$ at least continued after the same treatment program 18 to 24 months, adhere to the principle of combination therapy. The program includes at least 2 or 3 sensitive or untested anti-TB drugs. Intensive period is best composed of five drugs, the consolidation period of at least three kinds of drugs. Drug selection and 
program development should refer to the patient's previous medication history, drug sensitivity test results, adverse drug reactions and liver and kidney function and other comprehensive assessment, select the individual for the patient program.

\section{The Surgical Approach}

Surgery using intravenous anesthesia, double-lumen endotracheal intubation. (CVATS) or thoracoscopic assisted small incision (VAMT) surgery, according to the need for the implementation of pulmonary wedge resection, lobectomy or empty lesions removed surgery [ 26.27]. Surgery should follow the "maximum removal of lesions; as far as possible to retain lung tissue" principle. CVATS can be used two-hole method or three-hole method, with the progress of surgical techniques, in recent years, there have been thoracoscopic single hole surgery, 2012 by Gonzalez [28] first reported, soon introduced into the domestic, more than two holes Minimally invasive, the difficulty lies in the mutual interference of equipment, exposure is relatively difficult. Wang Haijiang et al [29] using thoracoscopic single hole surgery to treat tuberculosis with pneumothorax achieved satisfactory results. VAMT surgical incision length in the general about $10 \mathrm{~cm}$, Liao Yong, etc. [30] that, due to pleural adhesions, hilar lymph nodes and other reasons, VAMT become the primary choice for minimally invasive pulmonary tuberculosis surgery.

\section{Common Surgical Difficulties and Countermeasures}

Two-Dimensional Imaging. VATS is a two-dimensional imaging is not three-dimensional imaging, leading to surgical field of vision distortion, to determine the distance and depth of the organization more difficult, prone to side damage. In recent years, the emergence of 3D thoracoscopic surgery, Da Vinci surgical robotic system, to restore a similar thoracotomy surgery under the 3D field of vision, to improve the visual perception of the surgeon, the operation more accurate and safe [31.34].

Lung Laceration Is Hypoplasia. Leaf intermittent hypoplasia will increase the difficulty of VATS surgery, Pu Qiang [32] and so on using one-way lobectomy, laryngeal dysplasia is better. However, if there are hilar contracture, leaf lymph nodes, calcification, even with one-way lobectomy also have some difficulty, need to be changed to VMTA or conventional thoracotomy.

Pleural Adhesions. Most of the patients with pulmonary tuberculosis pleural adhesions, extensive pleural adhesions is a contraindication to thoracoscopic surgery. Thoracoscopy because of its wide field of vision, can be observed from different angles, for the treatment of local adhesions than thoracotomy has an advantage. For a wide range of adhesions, the fingers can be used between the two incision to open a pleural cavity to open a cavity, and then more extensive separation of thoracoscopy, separation should follow the principle of easy and difficult to encounter dense adhesion, should be from Loose place detour [33]. For the pleural cavity atresia, extensive adhesions lead to limitations of the endoscopic field of view, the operation of the hole with each other difficult to separate bleeding, time-consuming, very difficult to deal with, this situation should be decisive to give up simple thoracoscopic surgery, VMTA or conventional thoracotomy

Hilar Contracture, Hilar Vascular Anatomy Is Difficult. Pulmonary tuberculosis patients due to chronic lung infection, there will be hilar gutta, lymph node calcification, leading to pulmonary vascular anatomy difficult, VATS may damage the blood vessels lead to bleeding, a small amount of bleeding can be mirror through coagulation, suture, oppression, etc., There is difficult to control the bleeding, need to immediately turn the chest. So the VATS probe found hilar contracture, hilar vascular anatomy difficult situation, should not be reluctant to surgery, should be promptly changed to VMTA or conventional thoracotomy.

Surgery as an effective method of comprehensive treatment of tuberculosis is gradually being taken seriously, as long as the correct control of surgical indications, a reasonable choice of surgery, while strengthening the anti-TB treatment before and after treatment, the treatment effect is satisfactory. Minimally invasive surgery is the direction of development of surgery, thoracoscopic surgery for tuberculosis is more difficult compared to thoracic surgery, learning curve length, 
requiring the operator to be experienced, familiar with anatomy, and have a tacit understanding of the team. With advances in medical technology, the emergence of new technologies such as the 3D thoracoscopic system, the DaVinci Surgical Robot System [34] will push the minimally invasive surgical technique of thoracic surgery to a new level.

\section{References}

[1] Wang Sumin, Duanmu macro wish. The importance of strengthening tuberculosis control from drug - resistant tuberculosis [J]. Chinese Journal of General Practice Medicine, 2000, 3 (6): 488 489

[2] Wang Yanhe, Gong Youlong. Outbreak of tuberculosis in China, causes and influencing factors [J]. Journal of Medicine and Society, 2003, 16 (3): 7-14

[3] Li Wentao, Jiang Gening, Gao Wen, et al. Surgical treatment of 188 cases of multidrug-resistant tuberculosis [J]. Chinese Journal of Tuberculosis and Respiratory Diseases. 2006, 29: 524 - 526

[4] Yim AP, The role of video-assisted thoracosopic surgery in the management of pulmonary tuberculosis tu [J] .Chest, 1996,110 (3): 829-832.

[5] Kerti CA, Miron I, Cozma GV, et al. The role of surgery in management of pleuropul tu tuberculosis-seven years experience at a single institution [J] .Interact Cardiovasc thorac Surg, 2009, 8 (3): 334- 337.

[6] Kang MW, Kim HK, Choi YS, et al. Surgical treatment for multidrug-resistant and extensive drug-resistant tuberculosis [J]. Ann Thorac Surg, 2010, 89 (5): 1597-1602.

[7] Nie Tingsong, Sun Airong, Liu Jianglei, etc. TV thoracoscopic surgery in the treatment of pulmonary tuberculosis patients observed in 200 cases [J]. Chinese Journal of Medical Guide, 2010, 8 (23): 32

[8] Chen Haozhu. Practical Internal Medicine [M], People's Health Publishing Du. 1996: 78 - 79.

[9] Huang Zhiying, Zhou Mian, Lu Fudong. 50 cases of pulmonary tuberculosis patients with pathological type [J]. Chinese Journal of Tuberculosis and Respiratory Diseases, 1999, 22 (3): 153 155.

[10] Xie Boxiong, Ding Jiaan, Tang Mingjuan, et al. Diagnosis and surgical treatment of pulmonary aspergilloma [J]. Journal of Further Education, 2001.24 (10): 24 - 25.

[J]. Journal of Tuberculosis and Respiratory Diseases, 1984,7: 6 (in Chinese with English abstract)

[12] Zhang Lei, Ding Jiaan, Jiang Gening, etc. Pulmonary tuberculosis surgery indications [J] .2001,23 (5): 305 - 306

[13] Yang Hanqin. Elderly tuberculosis and lung cancer coexist 18 cases of clinical analysis [J]. Journal of Experimental Internal Medicine, 1998,11,572 - 573.

[14] Jiang Wenhang, Yuan Ping, Jiang Penghui, etc. Surgical treatment of pulmonary tuberculosis 670 cases [J]. Cardiopulmonary vascular disease, 2004,23 (4): 223 - 224.

[15] Bai Lianqi, Xu Shaofa. Progress in surgical treatment of tuberculosis [J]. Beijing Medical Association of thoracic surgery professional committee of the 2009 annual meeting of the Proceedings, 2009,12: 146 - 148.

[16] Wang Xiuxiu. Multidrug-resistant tuberculosis prevention [J]. Chinese Journal of Tuberculosis and Respiratory Diseases, 2006 (8): 511 - 513.

[17] BENJAMIN JP, JOSEPH CC, HEATHER K, et a1. Pulmonary resection for multi-drug resistant tuberculosis. Thoracic and Cardiovas Surg, 2001, 121 (3): 448 - 453. 
[18] Sun Shunxing, Cui Chao, Li Qiang. 98 cases of multi-drug tuberculosis surgical treatment of clinical analysis[J]. Tianjin Medical Journal, 2008, 36 (8): 639 - 641.

[19] van Leuven M, De Groot M, Shean KP, et a1. Pulmonary resection an adjunct in the treatment of multiple drug resistant tuberculosis. Ann Thorac Surg. 1997, 63: 1368-1373.

[20] Mohsen T, Zeid AA, Haj-Yahia S. Lobectomy or pneumoneetomy for muhidrug-resistant pulmonary tuberculosis can be performed by acceptable morbidity and mortality: a seven-year review of a single institution'B experience. J Thorac Cardiovaac Surg, 2007, 134: 194-198.

[21] Chen Qiliang, Guo Wanying, Yao Linming, et al. Clinical analysis of 186 cases of pulmonary tuberculosis treated by pneumonectomy [J]. Journal of Clinical Pulmonary Medicine, 2008, 13: 497.

[22] BENJAMIN JP, JOSEPH CC, HEATHER K, et a1. Pulmonaryresection for multi-drug resistant tuberculosis[J]. Thoracic and Cardiovas Surg, 2001, 121 f3): 448-453.

[23] Haifeng Wang, Hongsheng Lin, Gning Jiang. Pulmonary resection in the treatment of multidrug-resistant tubercu-losis: A retrospective study of 56 cases [J] Ann Thorac Surg, 2008, 86 (5): 1640-1645.

[24] Pomerantz BJ, Cleveland JC Jr, Olson HK, et al. Pulmonary resection for multi-drug resistant tuberculosis[J]. Thorac Cardiovasc Surg, 2001, 121: 448 - 453.

[25] Xue Zongxi, District Rui Gui, Zhao Binghang, etc. 89 cases of multi-drug-resistant pulmonary tuberculosis surgical treatment [J]. Guangdong Medical, 2002, 23 (11): 1177.

[26] Li Zhiqiang. Simple lung void removal in the treatment of severe pulmonary tuberculosis[J]. Guangxi Medical Journal, 2002,24 (10): 1565.

[27] Wei Li, etc.. Thoracoscopic hollow dissection for the treatment of empty tuberculosis[J]. Chinese Journal of Thoracic and Cardiovascular Surgery, 2004, 11 (2): 155-156.

[28] Gonzalez D, Paradela M, Garcia J, et al, Single-port video-assisted thoracoscopic lobectomy [J]. Interact Cardiovasc Thorac Surg, 2011, 12: 514-515.

[29] Wang Haijiang, Xia Zhaohua, Deng Guofang, etc. Single hole thoracoscopic surgery combined with pneumothorax pneumothorax clinical application. [J]. Journal of Clinical Pulmonary Medicine, 2016,24 (10): 1093-1095.

[30] Liao Yong, Wei Ming, Xu Jianrong, et al. Clinical analysis of video chest radiotherapy for thoracic tuberculosis [J]. Guangxi Medical Journal, 2012, 21 (6): 572-574.

[31] Qiao Wenliang, Zhou Jianhua, Liu Fenbing, etc.. 3D thoracoscopic system in the minimally invasive surgery in the chest of the initial application[J]. Chinese Journal of Cancer, 2015, 25 (4): 305-310.

[32] Pu Qiang, Liu Lunxu, car Guowei, et al. One-way full-thoracoscopic lobectomy for the treatment of lung benign disease $[\mathrm{J}]$. Journal of Sichuan University (Medical Science Edition), 2010,41 (3): 548-550.

[33] Che Yong, Liu Zhigang, Chang Wei, et al. Clinical analysis of 157 cases of thoracoscopic lobectomy [J]. Xinjiang Medical University, 2015, 45 (8): 1120-1126.

[34] Wang Shumin, Wang Chengfeng. Talking about the advantages and disadvantages of DaVinci robot surgery system and video-assisted thoracoscopic system in thoracic surgery lobectomy[J]. Journal of Clinical Medical Journal, 2016, 44 (6): 551-555. 